\title{
Do Children Have the Same Capacity to Perceive Affordances as Adults? An Investigation of Tool Selection and Use
}

\author{
Sara M. Scharoun \\ University of Waterloo \\ Pamela J. Bryden and Michael E. Cinelli \\ Wilfrid Laurier University \\ David A. Gonzalez and Eric A. Roy \\ University of Waterloo
}

\begin{abstract}
This study investigated whether 5- to 11-year-old children perceive affordances in the same way as adults $\left(M_{\text {age }}=22.93, S D=2.16\right)$ when presented with a task and four tools (nail in a block of wood and a hammer, rock, wrench, and comb; bucket of sand and a shovel, wooden block, rake, and tweezers; and a screw in a block of wood and a screwdriver, knife, dime, and crayon). Participants were asked to select the best tool and act on an object until all four assigned tools had been selected. No explicit instructions were provided because we were interested how task perception would influence tool selection and action. Results support the notion that the capacity to perceive affordances increases with age. Furthermore, differences in the way in which 5-year-olds acted on the screw in a block of wood demonstrated that the ability to detect some affordances takes longer to refine. Findings help to further the understanding of the development of perception-action coupling.
\end{abstract}

Keywords: children, motor development, affordances, perception-action

Proponents of the ecological perspective argue that perception is direct. Perceiving does not involve memory or representation; it is a process by which an animal detects useful information in its environment (Michaels \& Carello, 1981). The term affordance (J.J. Gibson, 1966, 1979; Michaels \& Carello, 1981; Turvey, 1992) is also used to describe the reciprocity between animal and environment, albeit with respect to action capabilities. According to the ecological view, an

Sara M. Scharoun, David A. Gonzalez, and Eric A. Roy are with the Department of Kinesiology, University of Waterloo, Waterloo, Ontario, Canada. Pamela J. Bryden and Michael E. Cinelli are with the Department of Kinesiology \& Physical Education, Wilfrid Laurier University, Waterloo, Ontario, Canada. Address author correspondence to Sara Scharoun at sscharou@uwaterloo.ca. 
animal perceives actions that are permitted (i.e., afforded) by objects, places, and events (J.J. Gibson, 1979). For example, we do not perceive a chair, we perceive a surface on which to sit—a surface that affords sitting.

As described by Michaels and Carello (1981), "The animal and environment fit together like interlocking pieces of a puzzle ... the animal fits into the environment but it is also the case that the environment fits around the animal" (p. 43). Affordances exist regardless of whether they are perceived or used. As such, an animal's ability to act in the environment, or use an affordance, is a process of information seeking based on action capabilities (Adolph, Eppler, \& Gibson, 1993). Returning to the example of the chair, the chair that affords sitting for an adult may afford a different action for a small child (e.g., support for standing). The compatibility between animal and environment with respect to action capabilities is referred to as an effectivity (e.g., Shaw, McIntyre, \& Mace, 1974; Shaw, Turvey, \& Mace, 1982).

Differences in the capacity to perceive affordances can be explained by perceptual and physical adaptations that occur with experience and growth. An animal is genetically preattuned to detect ecologically significant information in its environmental niche; however, survival ultimately depends on the acquisition of ecologically relevant knowledge (Adolph et al., 1993; Jacobs \& Michaels, 2007; Michaels \& Carello, 1981). It is argued that human infants perceive some affordances shortly after birth. However, they are unable to detect affordances that are available to adults because the biomechanical facets of the body limit the ability to act in the environment (Adolph, Weise, \& Marin, 2006; E.J. Gibson \& Walker, 1984). At birth, an infant's mobility is extremely limited. In turn, the ability to explore the environment is constrained. An infant quickly learns that crying elicits a caregiver response. Over the course of the 1st year, crying with an outstretched arm guides the caregiver to the object of an infant's interest. With the establishment of object manipulation, new affordances are exploited with changes in the ability of the actor to interact in his or her environment (Adolph et al., 1993).

The child learns what things are manipulable and how they can be manipulated, what things are hurtful, what things are edible, what things can be put together with other things or put inside other things — and so on without limit. He also learns what objects can be used as the means to obtain a goal, or to make other desirable objects, or to make people do what he wants them to do. (J.J. Gibson, 1966, p. 285)

Infants garner information from the environment through trial and error as their ability to act in the environment improves. As infants progress from creeping, to crawling, and eventually upright locomotion, the door for exploration opens into new spaces with different objects and surfaces to interact with (Smitsman \& Bongers, 2003; Smitsman \& Corbetta, 2010). The foundation for action gradually emerges. This enables infants to learn to detect new action possibilities (Adolph et al., 2006; E.J. Gibson \& Pick, 2000; Shaw \& Kinsella-Shaw, 1988), and the ability to attend to more useful affordances improves (Bongers, Smitsman, \& Michaels, 2004; Jacobs \& Michaels, 2007). With bodily growth, children are better able to overcome the degrees of freedom problem (Bernstein, 1967) associated with the tool-actor and tool-environment relationship. As a result, the capacity to perceive affordances becomes more adultlike with age. 
The current study sought to examine the capacity for children and adults to perceive the affordances of tools. This topic is of interest because the extent to which we use and create tools is a defining characteristic of the human species (Michaels \& Carello, 1981; Smitsman \& Bongers, 2003). Tools provide affordances for grasping and manipulating while also extending the ability to act effectively in the environment. Tools provide the opportunity to create new affordances for action (Adolph et al., 2006; J.J. Gibson, 1979; Smitsman, 1997; Wagman \& Carello, 2001). As expressed by J. J. Gibson (1979),

When in use, a tool is a sort of extension of the hand, almost an attachment to it or a part of the user's own body, and thus is no longer a part of the environment of the use. But when not in use, the tool is simply a detached object of the environment. . . . The boundary between the animal and the environment is not fixed at the surface of the skin but can shift. (p. 41)

The relationship between a tool and the environment, labeled tool-environment interface (Wagman \& Carello, 2003; Wagman \& Taylor, 2004) or topology (Smitsman \& Bongers, 2003; Smitsman, Cox, \& Bongers, 2006; Wagman \& Taylor, 2004), is one that is continuously evolving for humans. We manufacture complex tools, refine them on the basis of experience, make one tool to create another, and transfer knowledge to offspring over successive generations. Tool use is a characteristic trait of humans; therefore, it defines us as a species and separates us from our closest living relatives in the animal kingdom (Johnson-Frey, 2003). As summarized by Keen (2011), "The topic of tool use has never taken center stage in developmental psychology" (p. 3); however, it may help shed light on the development of action in humans (Lockman, 2000; Smitsman \& Bongers, 2003).

Tool use development has been described as problem of detecting a disconnect between one's ability to move and an action goal, recognizing that a tool can extend affordances, understanding topology, and using the tool successfully (Berger \& Adolph, 2003; Lockman, 2000; Smitsman, 1997; Smitsman \& Bongers, 2003). As demonstrated by McKenzie, Skouteris, Day, Hartman, and Yonas (1993), within the 1st year of life, the capacity to perceive affordances of tools changes quickly. In this study, seated infants were to reach for an object. By age 12 months, infants were able to perceive that their ability to reach was extended by the use of a tool, in this case a wooden spoon (McKenzie et al., 1993). The capacity to perceive the affordance of reachability improves infants' ability to act in their environment and aids in establishing independence.

Others have explored the perception of reachability with the aid of a tool in 2- to 4-year-olds, arguing that this age group uses more tools to explore and act; therefore, the capacity to perceive affordances will also change (Bongers, Smitsman, \& Michaels, 2004; Cox \& Smitsman, 2006a, 2006b; Smitsman, 1997). The study by Bongers et al. (2004) had children walk toward a table and displace a toy with a rod. Affordances were dependent on both rod length and the posture required to control the rod; therefore, the ability to perceive affordances was tied to learning to overcome the degrees of freedom necessary to control the rod. Because prospective control was observed more often in older ( 3 years, 3 months, to 4 years, 1 month) than younger ( 2 years, 2 months, to 3 years, 2 months) children, it was argued that, with age, children are better able to constrain the action system. That said, 
when comparing the results with those of a previous study with adults (Bongers et al., 2003), it was clear that the capacity for children to perceive affordances was not adultlike because children modified behavior on the basis of geometric but not kinetic properties (Bongers et al., 2004). In summary, results support the notion that, through development, we must learn to understand how the actor-tool relationship (the ability for an individual to act on a tool; e.g., ability to grasp a hammer) is constrained by the tool-environment relationship (the ability for a tool to act on an object; e.g., a hammer affords hitting a nail) to detect new affordances and act on them in tool use.

On the basis of this idea, the current study sought to examine the capacity for children and adults to perceive affordances in tool use. In other words, we aimed to investigate whether children and adults would perceive the actor-tool and tool-environment relationships in a similar manner. Participants were presented with four different tools and an object to act on (see Figure 1): (a) a hammer, rock, wrench, and comb were presented to act on a nail in a block of wood; (b) a shovel, wooden block, rake, and pair of tweezers to act on a bucket of sand; and (c) a screwdriver, knife, dime, and crayon to act on a screw in a block of wood. Tools
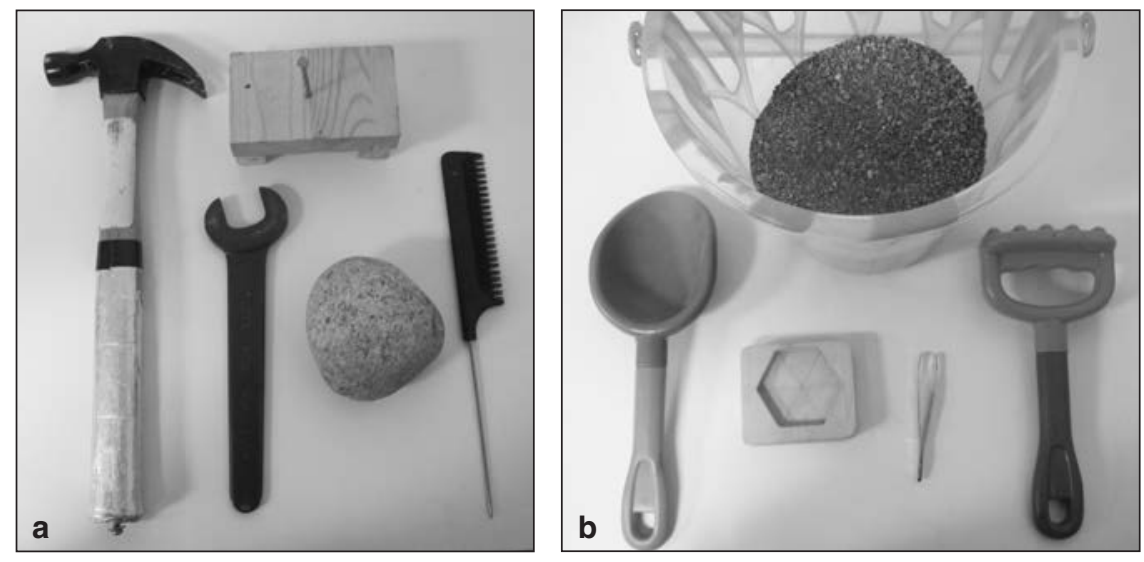

Figure 1 - Participants were asked to pick the best tool and act on an object: (a) hammer, rock, wrench, and comb and nail in a block of wood; (b) shovel, wooden block, tweezers, and rake and sand in a bucket; and (c) screwdriver, knife, dime, and crayon and screw in a block of wood.

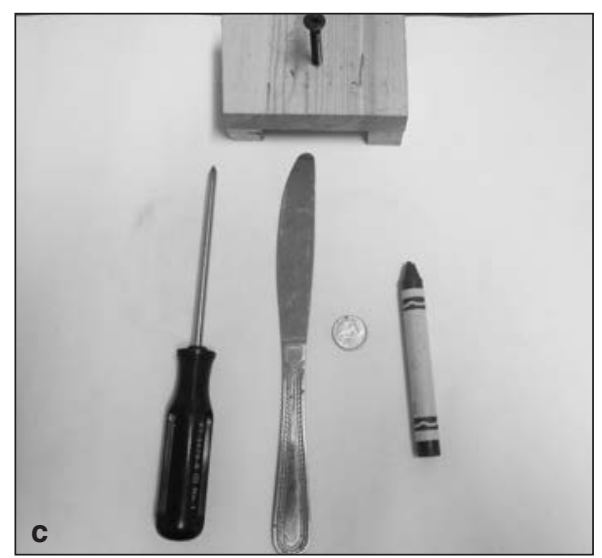


were selected by the researchers in consideration of similarities and differences in affordances. One tool (hammer, shovel, and screwdriver) was included because it was designed specifically to act on the object and thus offered the most linked affordances to act on the object. More specifically, a hammer affords hitting the nail, a shovel affords digging, and a screwdriver affords tightening or loosening the screw. Two tools were presented (rock, wrench, wooden block, rake, knife, and dime) because they offered similar affordances as the tool designed to act on the object. For example, the rock affords hitting the nail because of its hard surface, similar to the hammer. Finally, a nonsense tool (comb, tweezers, and crayon) was included as a distraction.

Participants were instructed to pick the best tool and act on the object presented, and no explicit instructions were provided. The ecological perspective states that affordances exist regardless of whether they are perceived or acted on; therefore, we were interested in what tasks would be perceived, how this would influence tool selection and action, and whether this would change across the ages studied (J.J. Gibson, 1979). Studies assessing affordances in infants (e.g., E.J. Gibson \& Walk, 1960; McKenzie et al., 1993) do not typically provide instructions, in part because of the inability to instruct infants and because an exploratory investigation is more in line with the ecological approach. The ability to act in the environment, or use an affordance, is a process of information seeking based on action capabilities (Adolph et al., 1993); therefore, not providing instructions enabled us to examine whether the tool-environment interface would override the actor-tool interface.

It was hypothesized that adults would attend to the most useful affordances, and tool selection would reflect perception of the tool that best afforded action. As such, it was expected that the hammer, shovel, and screwdriver would be selected first to perform the actions of hitting the nail, digging sand in the bucket, and tightening or loosening the screw, respectively. Furthermore, it was expected that the remaining three tools would be selected in an order that reflected perception of affordance-fit and the action would remain consistent throughout the duration of the study. It was hypothesized that the order in which tools would be selected and subsequent actions would become more adultlike with age because the capacity to detect useful information from the environment increases with age.

\section{Method}

\section{Participants}

Typically developing 5- to 11 -year-old children $(n=67)$ and a convenience sample of young adults from the university community $(n=14)$ participated in this study. The University Research Ethics Board approved all recruitment and testing procedures, and informed consent was obtained from all participants and parents or guardians of participating children. For means of comparison as a function of age, participants were separated into age groups: 5-year-olds $(n=14), 6$-year-olds $(n=$ 9), 7-year-olds $(n=19), 8$ - to 9-year-olds ( $n=11$; three age 9$), 10$ - to 11 -year-olds $(n=14$, two age 10$)$ and adults $\left(n=14, M_{\text {age }}=22.93\right.$ years, $\left.S D=2.16\right)$. Exact ages (i.e., in months) were not obtained for children. 


\section{Apparatus and Procedures}

The four tools were placed in front of the participant in peripersonal space, and the corresponding object was placed in peripheral space beyond the tools, along the midline (see Figure 1). Participants were not permitted to manipulate the tools or investigate the object before the study commenced. Four trials were completed with each object. Object and tool order was randomized to avoid an order effect. Participants were instructed to pick the best tool and act on the object presented. Instructions remained consistent for all participants throughout the duration of the study. For the first trial of each task, participants were asked, "Can you please select the best tool to complete the task?" In subsequent trials, participants were asked, "Can you please select the best tool to complete the task? You must choose a new tool." Participants were permitted to take their time in selecting a tool and acting on the object. If questions were asked, the instructions were repeated. A video camera was used to record tool selections and actions performed. These videos were coded offline.

\section{Data Analyses}

Videos were coded offline to note tool selection order (first, second, third, fourth), actions performed (1, whether they were adultlike, and 2, what the specific action was), and any changes in behavior (i.e., hesitating over a tool before selection or switching tool choice before action). Cohen's kappa was used to assess intrarater reliability. Levels of agreement were strong $(\geq 0.8)$ in the majority of cases; however, the level of agreement was good $(\geq 0.7)$ for tool selected fourth and action performed third in the screw-in-a-block-of-wood task and for and action performed second for the nail-in-a-block-of-wood task. Furthermore, the level of agreement was moderate $(\geq 0.6)$ for tool selected fourth in the screw-in-a-block-of-wood task and tool selected third for the nail-in-a-block-of-wood task.

Tool selection. Each object (nail in a block of wood, bucket of sand, and screw in a block of wood) was considered separately. The distribution of tools selected first, second, third, and fourth in each age group as a function of the total number of trials completed (i.e., four trials completed per tool-object combination) was computed. Fisher's exact tests using Age Group $\times$ Tool contingency tables were not feasible because of the numerous zero cells, as the assumption that all expected counts should be greater than 1 and no more than $20 \%$ of expected counts should be less than 5 was violated; therefore, statistical analyses were not feasible from this perspective (Field, 2013). Observations of tool selection were used to assess the capacity for children to perceive affordances. Kruskal-Wallis tests were used to evaluate differences in the proportion of trials (out of a total of four trials) for which the hammer, shovel, and screwdriver were selected first in each of the six independent age groups. Mann-Whitney $U$ tests were used for comparisons between the age groups.

Action. Each object (nail in a block of wood, bucket of sand, and screw in a block of wood) was considered separately, and specific actions were identified. Similar to analysis of tool selection order, Fisher's exact tests were not feasible, because assumptions of the statistical test were violated (Field, 2013). That said, 
all adults performed the action of hitting the nail, digging sand in the bucket, and tightening or loosening the screw. These observations were used to assess whether the task was perceived by children in the same way as by adults. Kruskal-Wallis tests were used to evaluate differences in the proportion of trials (out of a total of 16 trials) in which the action was adultlike (i.e., hammering the nail, digging sand in the bucket, tightening or loosening the screw). Despite clear instructions to select the most appropriate tool and subsequently act on the object, one 5-yearold, one 6-year-old, and two 7-year-olds did not perform an action with the tool. These children simply ranked the tools according to which was best to complete the task. Three 11-year-olds did not complete actions after the first trial of each task, stating that they would have performed the same action as in the previous trial. These children were removed from analysis. Mann-Whitney $U$ tests were used for comparisons between the age groups.

Changes in behavior. As mentioned earlier, participants were not permitted to manipulate the tools or investigate the object before the study commenced. However, after the study commenced (i.e., once instructions were provided), participants were permitted to take their time in selecting a tool and acting on the object. As such, changes in behavior (hesitating over a tool before selection or switching tool choice before action) were observed and recorded. It is important to note that this did not interfere with identification of tool selection order, because the final tool selected to complete the task was recorded in each trial. In the case of participants who did not act on the object, no hesitation or changes in selection were observed. We sought to perform Fisher's exact tests using Age Group 'Behavior contingency tables; however, this was not feasible because the assumptions of the statistical test were once again violated (Field, 2013).

\section{Results}

\section{Tool Selection}

The distributions of tools selected are displayed in Figures 2, 3, and 4. As depicted, $100 \%$ of adults selected the hammer first when acting on the nail in a block of wood (see Figure 2) and the shovel first when acting on the bucket of sand (see Figure 3 ). In addition, all but one adult (who selected the knife in three of four trials) selected the screwdriver first when acting on the screw in a block of wood (see Figure 4).

There was a significant effect of age on selecting the hammer first to act on the nail in a block of wood, $H(5)=19.382, p=.002$ (see Figure 5). Adults selected the hammer first significantly more often than 5- $(U=28.0, p<.001), 6-(U=35.0, p$ $=.008), 7$ - $(U=91.0, p=.023)$, and 8- to 9-year-olds $(U=56.0, p=.042)$. There was no difference between 10 - to 11 -year-olds and adults $(U=84.0, p=.15)$. Observing differences that emerged in children, 5-year-olds selected the hammer first significantly less often than 7- $(U=72.5, p=.017), 8$ - to 9 - $(U=38.5, p=$ $.024)$, and 10- to 11-year-olds $(U=46.0, p=.008)$.

A significant effect of age on selecting the shovel first to act on the bucket of sand was also revealed, $H(5)=27.701, p<.001$ (see Figure 5). Adults selected the shovel first more often than 5- $(U=21.0, p<.001), 6-(U=35.0, p=.007)$, and 7 -year-olds $(U=77.0, p=.006)$. No differences emerged between adults and 8 - to 


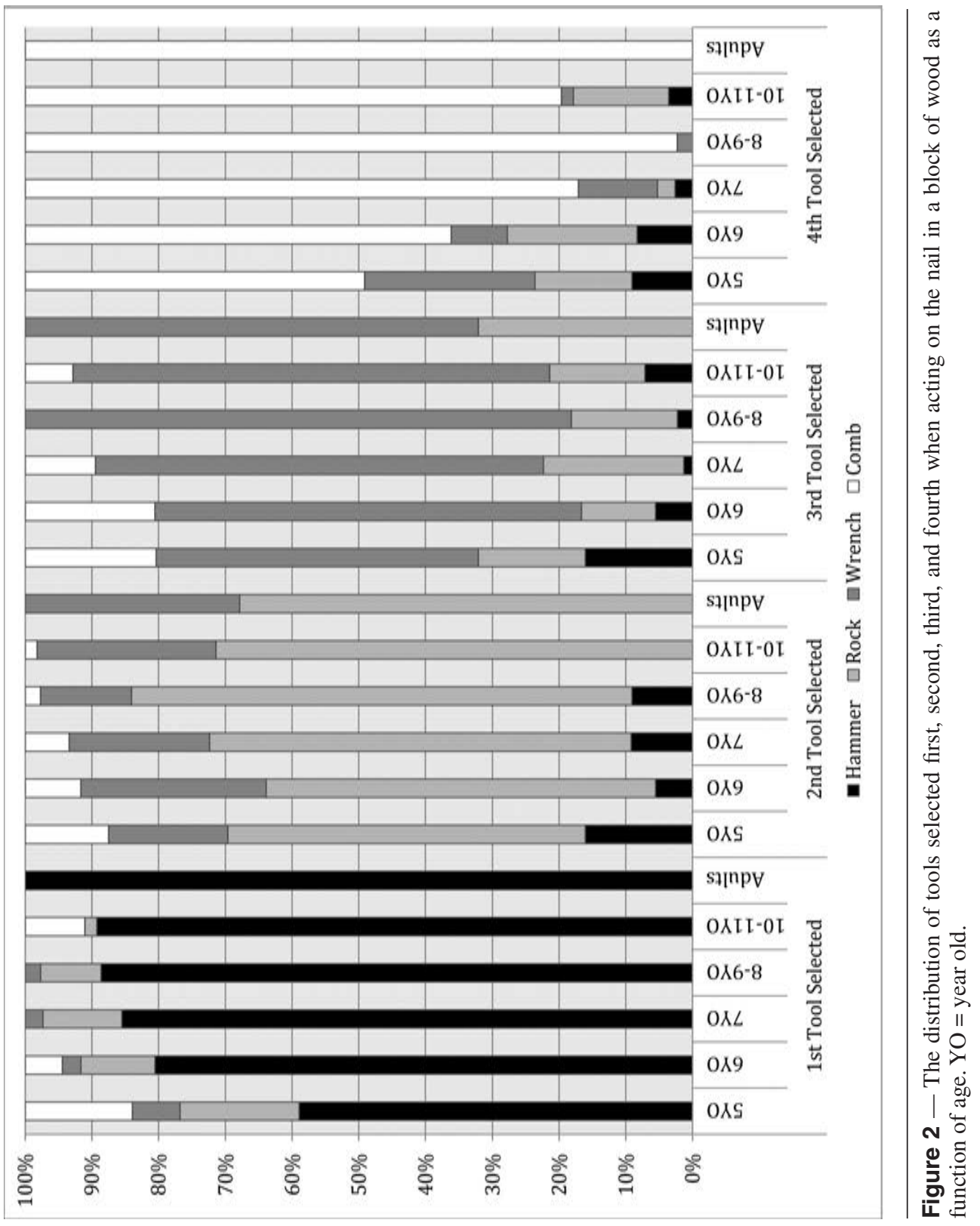




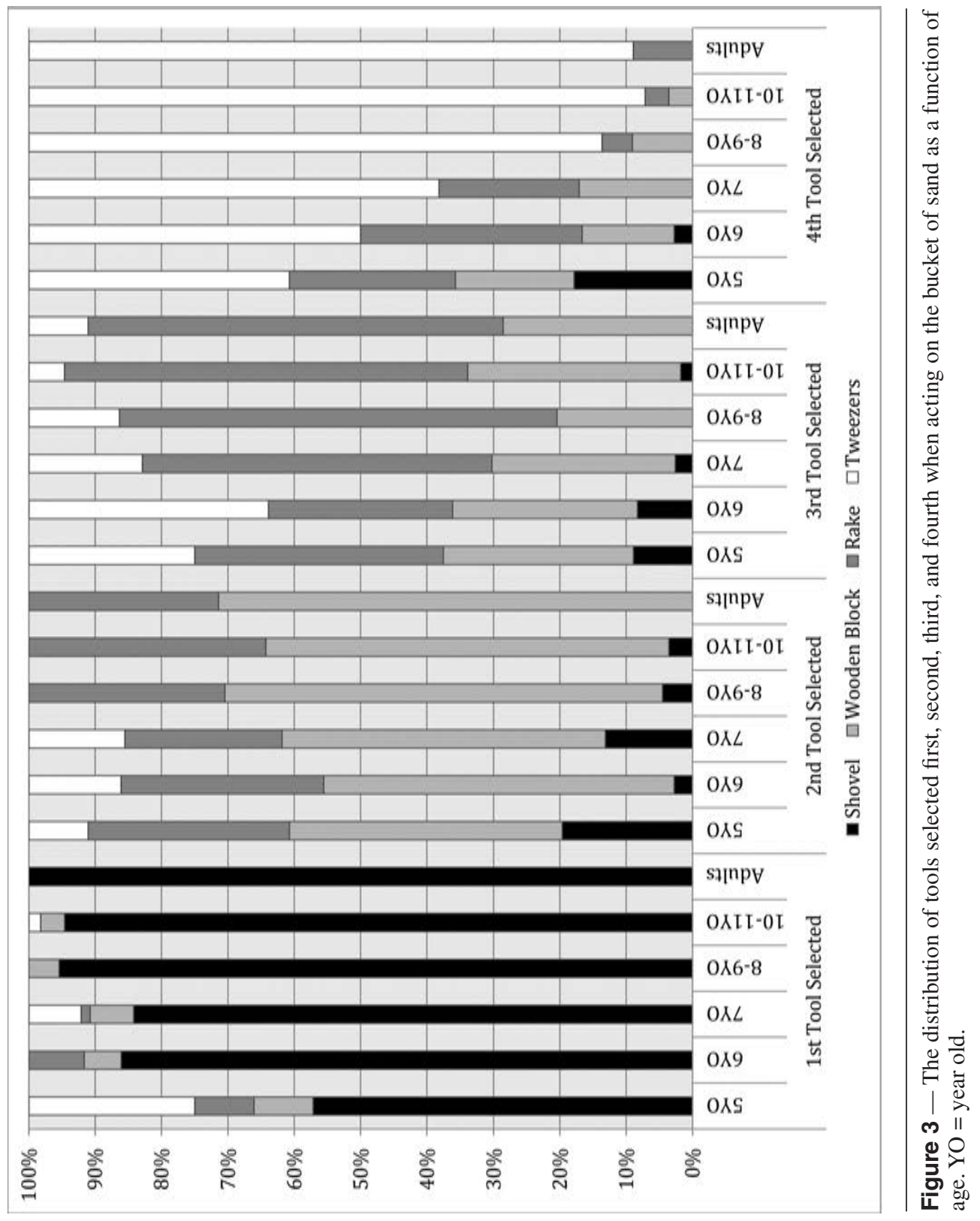




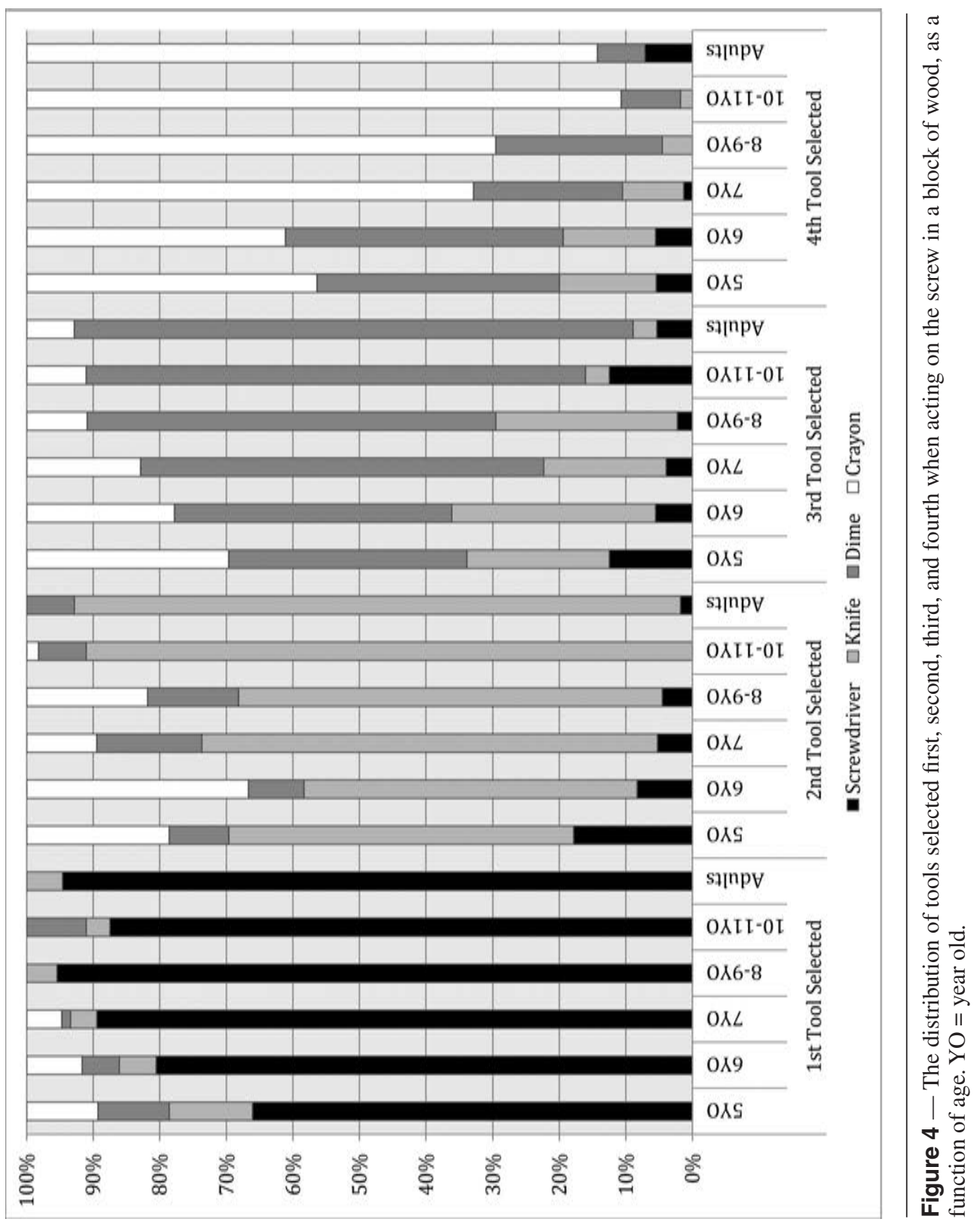




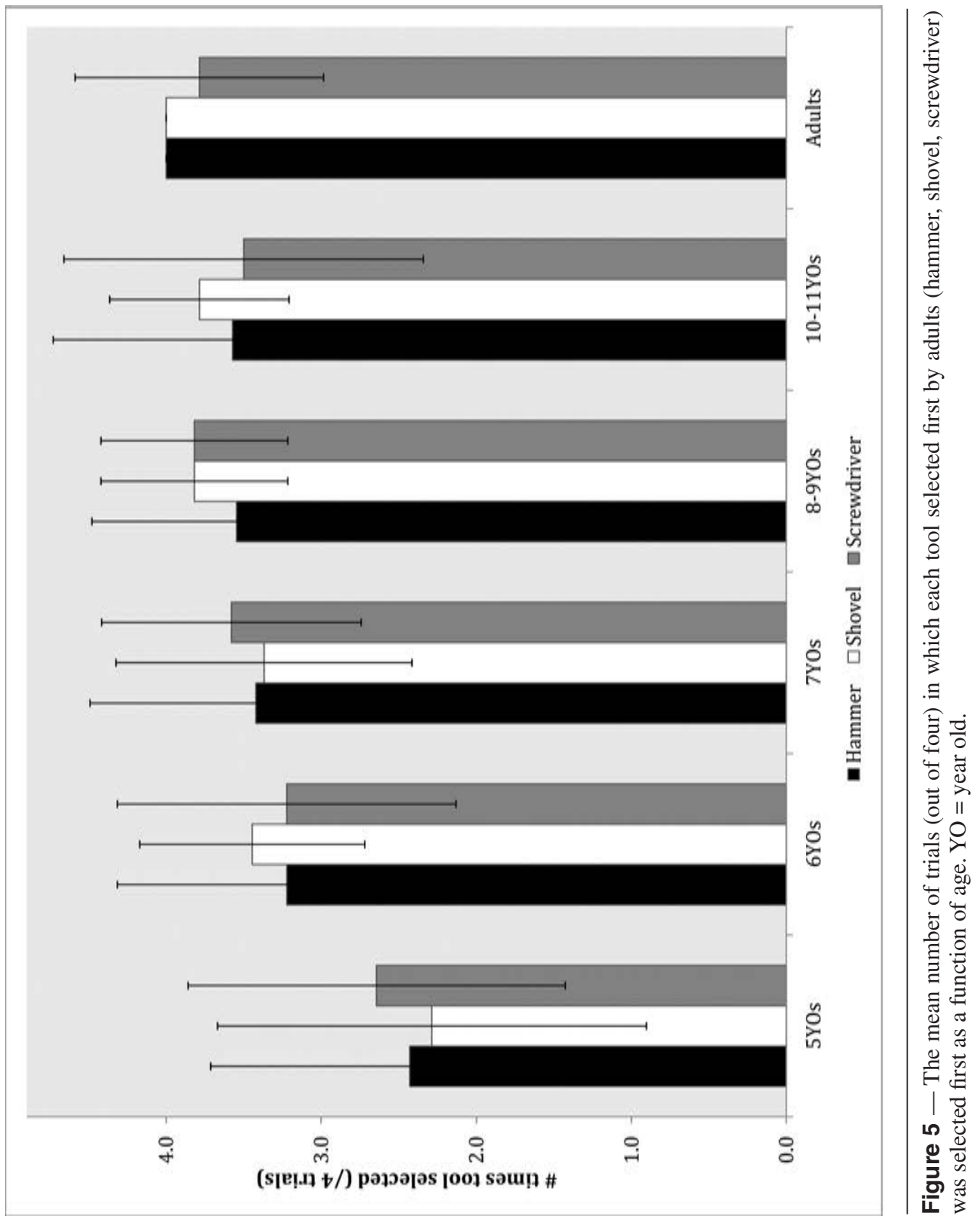


9- $(U=70.0, p=.259)$ and 10 - to 11 -year-olds $(U=84.0, p=.150)$. Observing differences that emerged in children, 5-year-olds selected the shovel first significantly less often than 6- $(U=31.0, p=.036), 7-(U=68.5, p=.013), 8$ - to 9- $(U=23.5$, $p=.002)$, and 10- to 11-year-olds $(U=31.5, p=.001)$.

Finally, there was a significant effect of age on selecting the screwdriver first to act upon the screw in a block of wood $(H(5)=17.245, p=.004$; see Figure $5)$. Adults selected the screwdriver significantly more often than 5-year-olds. No differences emerged between adults and 6- to 11-year-olds. Observing differences that emerged in children, 5-year-olds selected the screwdriver first significantly less often than 7- $(U=68.5, p=.010), 8$ - to $9-(U=30.0, p=.004)$, and 10 - to 11 -year-olds $(U=51.5, p=.019)$.

\section{Action}

For the nail in a block of wood, actions included hitting the nail, placing the tool on the nail (i.e., balancing it), and a turning motion (with the wrench). For the bucket of sand, actions included digging and, for one child, filling the wood block with dirt. Finally, for the screw in a block of wood, actions included tightening or loosening the screw, placing the tool on the screw (i.e., balancing), coloring (with the crayon), cutting (with the knife), and hitting the screw. The distributions of actions performed as a function of age are displayed in Figures 6-8.

There was no effect of age on performing the action of digging, $H(5)=4.692$, $p=.455$. However, an effect of age on performing the action of hammering, $H(5)$ $=14.346, p=.014$, revealed 6-year-olds performed the action less than young adults $(U=21.0, p=.016)$. An effect of age on performing the action of tightening or loosening the screw also emerged, $H(5)=19.121, p=.002)$. Mann-Whitney $U$ tests revealed adults performed the action of tightening or loosening the screw significantly more often than 5-year-olds $(U=35.0, p=.006)$. Observing differences that emerged in children, 5-year-olds performed the action of tightening or loosening the screw significantly less often than 7- $(U=58.5, p=.023)$ and 8-to 9-year-olds $(U=31.5, p=.018)$.

\section{Changes in Behavior}

The distributions of behaviors (consistent, hesitated before selection, and switched tool after selection) are displayed in Figure 9.

\section{Discussion}

The present study examined the capacity for children and adults to perceive affordances in tool use. More specifically, we aimed to investigate whether children and adults would perceive the actor-tool and tool-environment relationships in a similar manner, as assessed by tool selection and action. It was hypothesized that adults' tool selection would reflect perception of the task (i.e., tool-environment fit); therefore, the tool that best afforded action would be selected first, where subsequent selection would be based around the action capabilities of the remaining tools (i.e., tool-object fit). In line with our hypothesis, the hammer, shovel, and screwdriver were selected first by adults to perform the actions of hitting the nail, 


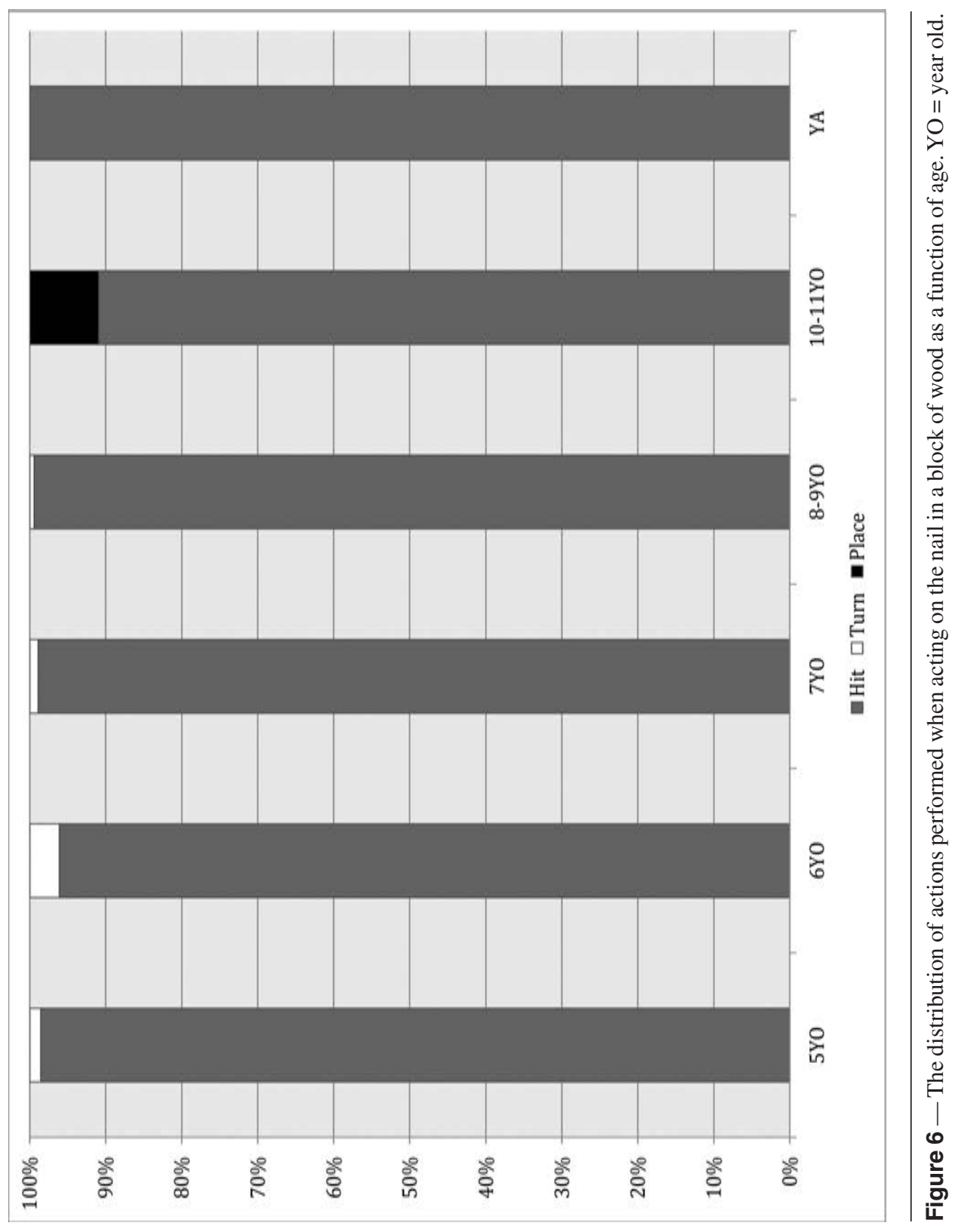




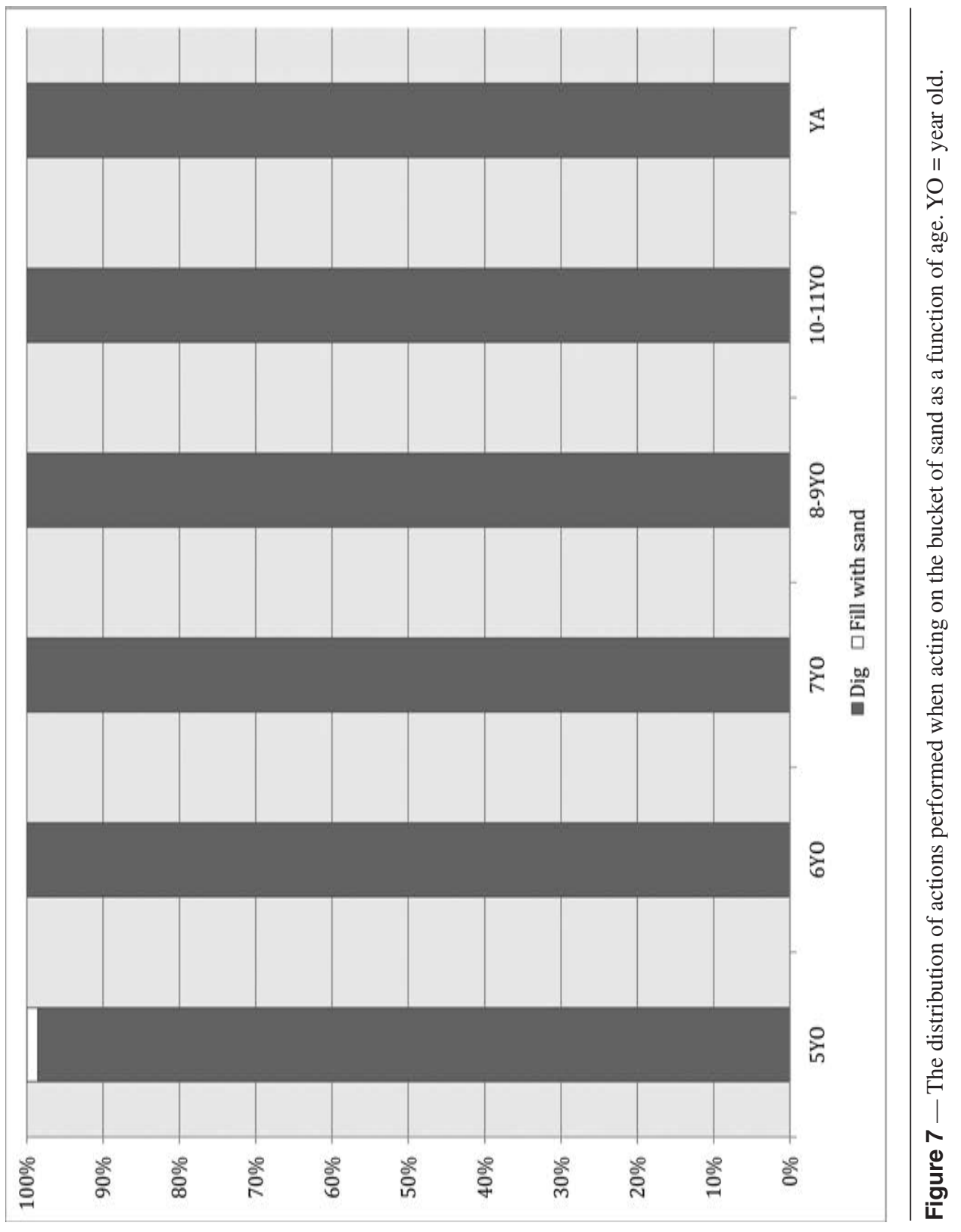




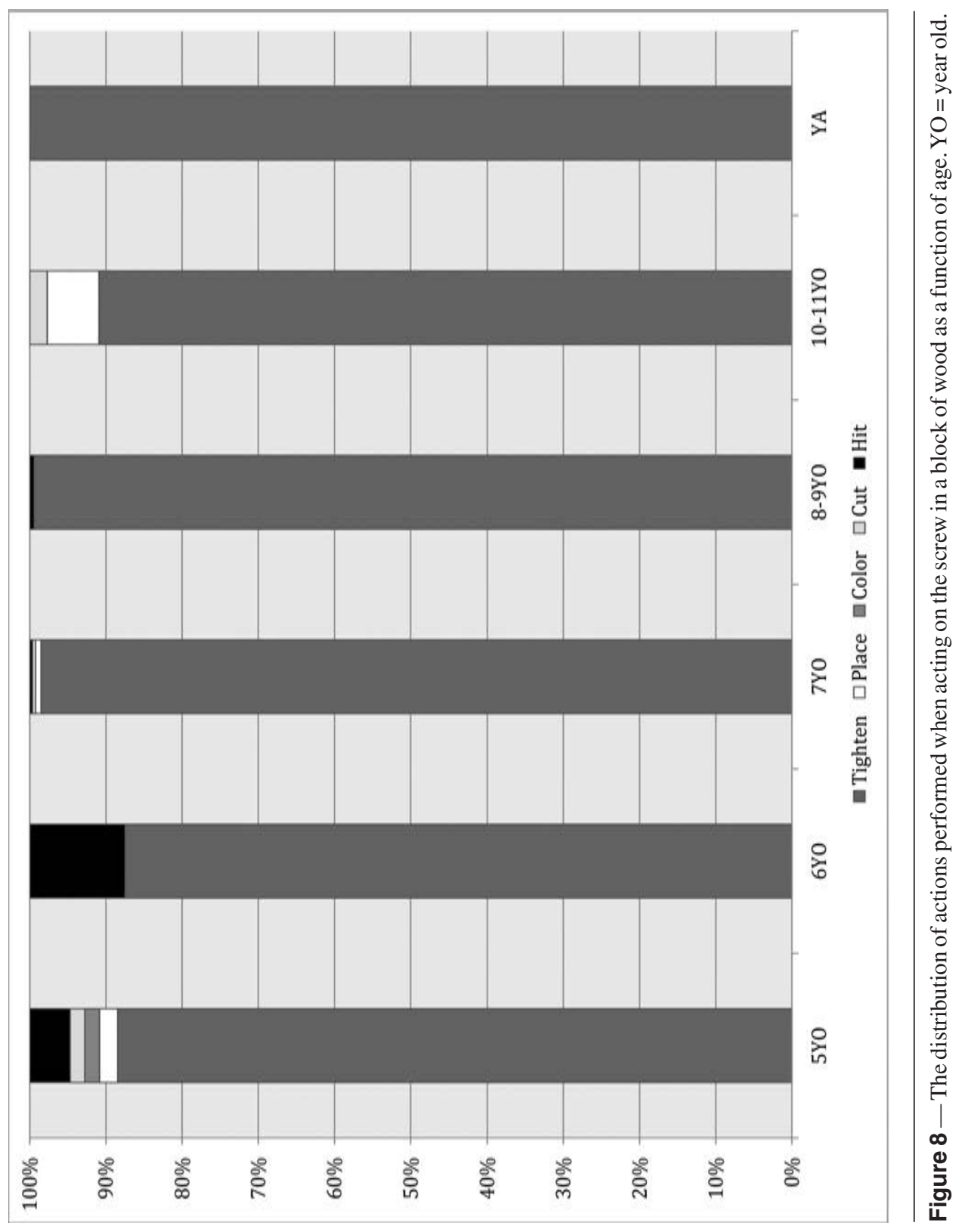




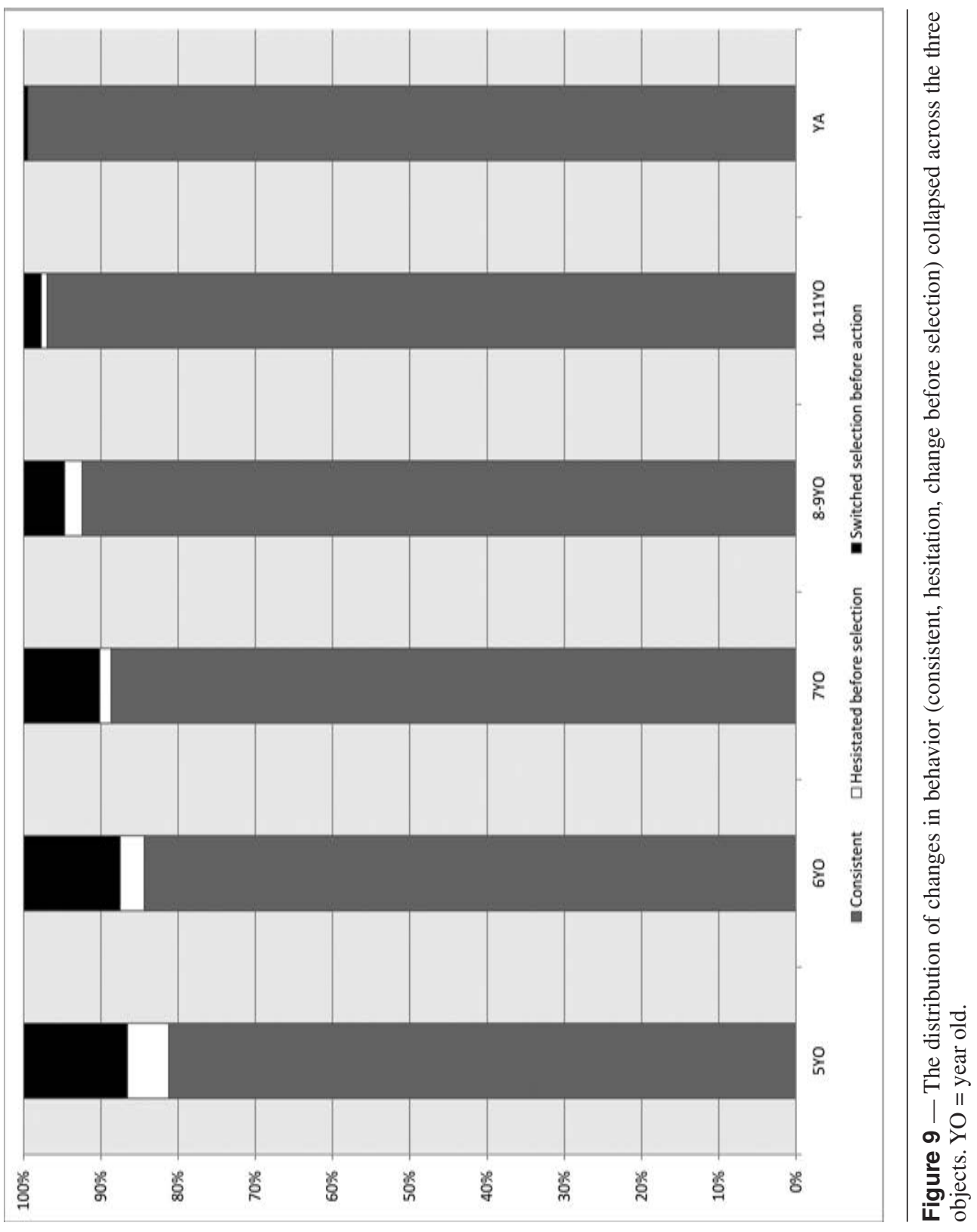


digging sand in the bucket, and tightening or loosening the screw, respectively. Tool selection and action in adults were used as a basis for comparison with children. Results are discussed in turn.

\section{Tool Selection}

It was expected that the capacity to perceive affordances, as evidenced in tool selection and action, would become more adultlike with increasing age in the children. More specifically, we hypothesized that tool selection and action in young children would reflect learning to detect useful information in the environment through trial and error, and thus detection of the affordance fit of each tool would be refined with age. Our hypotheses were partially supported, such that a significant effect of age on tool selection was observed; however, the extent differed as a function of toolobject combination. With the nail in a block of wood, 5- to 9-year-olds selected the hammer first less often than adults. In addition, 5-year-olds selected the hammer first less often than 7- to 11-year-olds. With the bucket of sand, 5- to 7-year-olds selected the shovel first less frequently than adults; likewise, 5-year-olds selected the shovel first significantly less often than 6- to 11-year-olds. Finally, with the screw in a block of wood, 5-year-olds selected the screwdriver significantly less frequently than 6- to 11-year-olds and adults.

It is clear that the capacity for 5-year-olds to perceive the affordances of tools is significantly different than that of older children and adults and that the capacity to perceive affordances increases with age in children. As displayed in Figures 2, 3 , and 4, the 5-year-olds did select the same tool as adults at a level that was above chance (i.e., $>50 \%$ ). Furthermore, similarities in action with a nail in a block of wood and a bucket of sand were observed and support the notion that the 5-year-olds did perceive the same action but selected tools on the basis of different action capabilities.

When acting with a tool, the tool becomes an extension of the body, therefore extending the action system and creating new affordances for action (J.J. Gibson, 1979). To act using affordances, one must learn to overcome the degrees-of-freedom problem (Bernstein, 1967) inherent in the tool-actor relationship to control the tool while simultaneously understanding the tool-environment relationship to act on the object (e.g., Bongers et al., 2004). The ability to do so is limited by bodily growth and prospective control, which undoubtedly influences a developing child's capacity to perceive affordances (e.g., Bongers et al., 2004; Jacobs \& Michaels, 2007). Learning involves discriminating actions that are possible from those that are not (Adolph et al., 2006). It is thus likely that children in this study-especially 5-yearolds-were exploring the tool-environment relationship to decipher which tool could be better controlled (i.e., better afforded grasping and manipulation) and therefore could better afford acting on the object. It is likely that children may have continued to explore the tools' affordances if they had been given more time to explore the tasks. Furthermore, if given an object to hold ahead of time and then asked to act, children may have acted differently, because they would have been exploring the actor-tool relationship before being exposed to the tool-environment relationship.

It is through exploration that children learn about object properties and how to refine their own actions. The notion of exploring was further demonstrated when examining changes in behavior. Although we were unable to analyze the data statistically, it is clear from Figure 9 that young adults were consistent in their selection 
tendencies. In other words, they did not hesitate over one tool before selection, and they did not switch their tool selection before acting on the object. Observations of children revealed that, despite consistent selection at least $80 \%$ of the time, there was evidence of hesitation and tool switches before action, which decreased as a function of age. This was likely due to familiarity with the task or tool.

Anecdotal reports also provide support for explorative action. Specifically, 5-year-olds described that the rock was selected first because it was "the best" (Participant 5) and it "works better" (Participant 10). It has been suggested that preschoolers are in a phase of discovery, in which they are exploring "how best to use perceptual information to calibrate the motor system in the service of action" (Fitzpatrick, Wagman, \& Schmidt, 2012, p. 28). As a result, selection appeared more adultlike with age.

It is generally understood that perceptual abilities become better refined and more differentiated with age as children garner knowledge through exploring (E.J. Gibson, 2000). This is exemplified anecdotally in the current study. Some of the 5-, 6-, and 7-year-olds (although fewer 7-year-olds) claimed to be unfamiliar with some of the tools. From the perspective of the researchers, it can be argued these children were, therefore, more inclined to explore their affordances. For example, the tweezers were a novel tool for some of the children. One 5-year-old expressed, as she picked the tweezers first, knowing that they were not the best tool, but that she "just wanted to try them" (Participant 4). Consequently, it can be suggested that the younger children in particular were curious as to what action the tweezers afforded. Therefore, it is likely that children altered their tool selection to allow for comparison of the affordance fit of the tools through trial and error.

Children (all age groups) also discussed how the shovel was best suited to pick up a lot of dirt; however, if one only wanted a small amount of sand, or a pebble, the tweezers were best suited. For example, one 5-year-old child described that picking the tweezers first because "they pick little things up easier" (Participant 10). This anecdotal evidence, in conjunction with the tool selection order of young children, provides further support for the suggestion that children aimed to delineate the affordance fit of each tool (E.J. Gibson, 2000) and, furthermore, that the perception of action altered the order in which tools were selected.

\section{Action}

No effect of age emerged for action on the nail in a block of wood or bucket of sand. Similar to adults, children perceived the tools presented to afford hitting the nail in the block of wood and digging in the bucket of sand. Interestingly, this was not the case for the screw in a block of wood, where an effect of age was evident. More specifically, 5-year-olds tightened or loosened the screw significantly less often than 7- to 8-year-olds and adults. These children were observed hitting the nail with the screwdriver, cutting the block of wood with the knife, placing the dime on the screw, and coloring on the block of wood with the crayon. In addition, one 11-year-old balanced (i.e., placed) each of the tools on the screw, which explains why the actions of 5- and 10- to 11-year-olds did not differ.

The fact that differences as a function of age were found exclusively when acting on the screw in a block of wood suggests that, in comparison with acting on the nail in a block of wood and bucket of dirt, a different tool-environment relationship was 
perceived by children. In line with this idea, it can be argued that the affordances of tightening or loosening the screw in the block of wood were not as easily detected. The difference in actions between the children and the adults emerged because a screw and screwdriver-like tools do not occur naturally in the environment; they are manmade and therefore their proper use is based more on experience and exposure than on affordances. Furthermore, it is important to consider the fine motor precision required to match the tip of a screwdriver with the head of a screw; therefore, the affordance fit may have been more difficult to perceive. As a result, children acted on other useful information, which was by no means wrong, because the task was not explicitly stated, just different on the basis of perception of the task. The screw, similar to the nail, has expanded the human ability to build and construct. Put simply, the nail and screw differ solely on the basis of the absence (nail) or presence (screw) of threading. However, it is these minute differences that change the affordance fit defining the actor-tool and tool-environment relationships. To act on a nail affords hitting, whereas to act on a screw affords tightening or loosening. Differences in affordances also lead to differences in tool-environment fit, such that a nail can arguably be hit by any tool that offers a hard surface, whereas to tighten or loosen a screw requires a specific match between tool (i.e., screwdriver or, in this case, knife and dime) and screw. As expressed by Michaels and Carello (1981),

the acting animal must have a specification of "tuning" parameters that adapt the details of motor activities to the situation at hand. While both level and sloping group may afford walking, the manner in which that gait is executed must be geared to the slope of the surface. Thus, the domain of the perceptionaction interrelationship includes not only global aspects (a ball affords throwing) but also minute ones (a baseball affords throwing in a different way from a whiffle ball). (p. 48)

An object such as a screw thus requires a greater capacity to detect useful information and perceive affordances based on minute aspects of the actor-environment fit.

\section{Summary and Conclusions}

Overall, findings of the current study are in line with previous accounts of perceptual learning (E.J. Gibson, 2000). Learning through exploration allows children to garner information about the manner in which a tool or action changes their environment, thus delineating affordances. Eleanor J. Gibson (2000) stated that an "activity that starts as exploratory can become performatory as an affordance is discovered" (p. 297). As such, 5-year-old children in the current study were the least adultlike in their capacity to perceive affordances. As exemplified with the screw in a block of wood, some affordances are not as easily perceived. Nonetheless, with age and acquired motor experience, as displayed by older children, the capacity to perceive affordances becomes refined and better differentiated, and bodily growth enables children to increase action capabilities (E.J. Gibson, 2000). These findings have important implications for our understanding of tool use development in humans, because they may help to further our understanding of the development of perception-action coupling and the extent to which it separates us from other species. 


\section{References}

Adolph, K.E., Eppler, M.A., \& Gibson, E.J. (1993). Development of perception of affordances. In C. Rovee-Collier, L.P. Lipsitt, \& H. Hayne (Eds.), Advances in infancy research (Vol. 8, pp. 51-98). Santa Barbara, CA: Praeger.

Adolph, K.E., Weise, I., \& Marin, L. (2006). Motor development. In K.E. Adolph \& S.E. Berger, Encyclopedia of cognitive science. doi:10.1002/0470018860.s00584

Berger, S.E., \& Adolph, K.E. (2003). Infants use handrails as tools in a locomotor task. Developmental Psychology, 39, 594-605. doi:10.1037/0012-1649.39.3.594

Bernstein, N. (1967). The co-ordination and regulation of movements. Oxford, England: Pergamon Press.

Bongers, R.M., Smitsman, A.W., \& Michaels, C.F. (2003). Geometries and dynamics of a rod determine how it is used for reaching. Journal of Motor Behavior, 35, 4-22. doi:10.1080/00222890309602117

Bongers, R.M., Smitsman, A.W., \& Michaels, C.F. (2004). Geometric, but not kinetic, properties of tools affect the affordances perceived by toddlers. Ecological Psychology, 16, 129-158. doi:10.1207/s15326969eco1602_3

Cox, R.F., \& Smitsman, W. (2006a). Action planning in young children's tool use. Developmental Science, 9, 628-641. doi:10.1111/j.1467-7687.2006.00541.x

Cox, R.F., \& Smitsman, A.W. (2006b). The planning of tool-to-object relations in young children. Developmental Psychobiology, 48, 178-186. doi:10.1002/dev.20117

Field, A. (2013). Discovering statistics using IBM SPSS statistics (4th ed.). Thousand Oaks, CA: Sage.

Fitzpatrick, P., Wagman, J.B., \& Schmidt, R.C. (2012). Alterations in movement dynamics in a tool-use task: The role of action-relevant inertial tool properties. Zeitschrift fur Psychologie mit Zeitschrift fur Angewandte Psychologie, 220(1), 23-28. doi:10.1027/2151-2604/a000087

Gibson, E.J. (2000). Perceptual learning in development: Some basic concepts. Ecological Psychology, 12, 295-302. doi:10.1207/S15326969ECO1204_04

Gibson, E.J., \& Pick, A.D. (2000). An ecological approach to perceptual learning and development. New York, NY: Oxford University Press.

Gibson, E.J., \& Walker, A.S. (1984). Development of knowledge of visual-tactual affordances of substance. Child Development, 55, 453-460. doi:10.2307/1129956

Gibson, J.J. (1966). The senses considered as perceptual systems. Boston, MA: Houghton Mifflin.

Gibson, J.J. (1979). The ecological approach to visual perception. Boston, MA: Houghton Mifflin.

Jacobs, D.M., \& Michaels, C.F. (2007). Direct learning. Ecological Psychology, 19, 321-349. doi:10.1080/10407410701432337

Johnson-Frey, S.H. (2003). What's so special about human tool use? Neuron, 39, 201-204. doi:10.1016/S0896-6273(03)00424-0

Keen, R. (2011). The development of problem solving in young children: A critical cognitive skill. Annual Review of Psychology, 62, 1-21. doi:10.1146/annurev. psych.031809.130730

Lockman, J.J. (2000). A perception-action perspective on tool use development. Child Development, 71, 137-144. doi:10.1111/1467-8624.00127

McKenzie, B.E., Skouteris, H., Day, R.H., Hartman, B., \& Yonas, A. (1993). Effective action by infants to contact objects by reaching and leaning. Child Development, 64, 415-429. doi:10.2307/1131259

Michaels, C.F., \& Carello, C. (1981). Direct perception. Englewood Cliffs, NJ: PrenticeHall. 
Shaw, R., \& Kinsella-Shaw, J. (1988). Ecological mechanics: A physical geometry for intentional constraints. Human Movement Science, 7, 155-200. doi:10.1016/01679457(88)90011-5

Shaw, R., McIntyre, M., \& Mace, W. (1974). The role of symmetry in event perception. In R. B. MacLeod \& H. L. Pick (Eds.), Perception: Essays in honor of James J. Gibson (p. 317). Ithaca, NY: Cornell University Press.

Shaw, R., Turvey, M. T., \& Mace, W. (1982). Ecological psychology: The consequence of a commitment to realism. Cognition and the Symbolic Processes, 2, 159-226.

Smitsman, A. W. (1997). The development of tool use: Changing boundaries between organism and environment. In C. Dent-Read \& P. Zukow-Goldring (Eds.), Evolving explanations of development: Ecological approaches to organism-environment systems (pp. 301-329). doi:10.1037/10265-008

Smitsman, A., \& Bongers, R. (2003). Tool use and tool making: A developmental action perspective. In J. Valsiner \& K.J. Connolly (Eds.), Handbook of developmental psychology (pp. 172-193). doi:10.4135/9781848608306.n8

Smitsman, A.W., \& Corbetta, D. (2010). Action in infancy-Perspectives, concepts and challenges. In J.G. Bremner \& T.D. Wachs (Eds.), The Wiley-Blackwell handbook of infant development (2nd ed., Vol. 1, pp. 167-203). doi:10.1002/9781444327564.ch5

Smitsman, A.W., Cox, R.F.A., \& Bongers, R.M. (2006). Action dynamics in tool use. In V. Roux \& B. Bril (Eds.), Stone knapping: The necessary conditions for a uniquely hominin behaviour (pp. 129-147). Cambridge, England: McDonald Institute for Archaeological Research.

Turvey, M.T. (1992). Affordances and prospective control: An outline of the ontology. Ecological Psychology, 4, 173-187. doi:10.1207/s15326969eco0403_3

Wagman, J. B., \& Carello, C. (2003). Haptically creating affordances: the user-tool interface. Journal of Experimental Psychology: Applied, 9(3), 175-186. doi:10.1037/1076898X.9.3.175

Wagman, J.B., \& Carello, C. (2001). Affordances and inertial constraints on tool use. Ecological Psychology, 13, 173-195. doi:10.1207/S15326969ECO1303_1

Wagman, J.B., \& Taylor, K.R. (2004). Chosen striking location and the user-toolenvironment system. Journal of Experimental Psychology. Applied, 10, 267-280. doi:10.1037/1076-898X.10.4.267 